\title{
Circuit
}

Musiques contemporaines

\section{Souvenirs vivants de Mario Bertoncini}

\section{Charles De Mestral}

Volume 15, numéro 1, 2004

Interpréter la musique (d')aujourd'hui

URI : https://id.erudit.org/iderudit/902345ar

DOI : https://doi.org/10.7202/902345ar

Aller au sommaire du numéro

Éditeur(s)

Les Presses de l'Université de Montréal

ISSN

1183-1693 (imprimé)

1488-9692 (numérique)

Découvrir la revue

Citer ce document

De Mestral, C. (2004). Souvenirs vivants de Mario Bertoncini. Circuit, 15(1),

93-95. https://doi.org/10.7202/902345ar d'utilisation que vous pouvez consulter en ligne.

https://apropos.erudit.org/fr/usagers/politique-dutilisation/ 


\section{Souvenirs vivants de Mario Bertoncini}

par Charles de Mestral

Mario Bertoncini a offert un atelier de design musical pendant les années scolaires 1975-1976 et 1976-1977 à la Faculté de musique de l'Université McGill. Les étudiants qui s'étaient inscrits ne se doutaient pas que leur vie musicale en serait radicalement transformée. Dans un contexte scolaire, nécessairement un peu formaliste, l'arrivée de Bertoncini était une bouffée de vie. II ne se présentait pas seulement comme professeur mais comme artiste. II nous a montré par l'exemple comment être artistes-musiciens à la fin du xxe siècle.

On s'est retrouvés dans un atelier d'un genre inattendu avec deux pôles de travail, l'improvisation musicale ainsi que le travail de conception et de réalisation de "sources sonores" électroacoustiques nécessitées par la composition musicale. En premier lieu, il avait apporté avec lui tout un ensemble d'instruments musicaux et de sources sonores : un tam-tam, des gongs chinois, des cymbales, des blocs de bois, des flûtes, des appeaux, des archets pour le piano de son invention, des sifflets, ses propres harpes éoliennes à air comprimé... ainsi que des moyens d'amplification, de filtrage et de modification sonores électroacoustiques.
II nous a plongés dans le contexte d'un atelier d'improvisation sonore, partageant avec nous une partie de son expérience approfondie à travers le travail du groupe Nuova Consonanza. II nous a mis au défi de la recherche et de la création de mondes sonores originaux. Ses consignes étaient surtout négatives : pas de structures harmoniques ou mélodiques évidentes, pas de rythmes périodiques, pas de formules ressemblant à un style musical ancien ou réputé d'avant-garde. Que restet-il quand, conscient de la tradition, on s'impose radicalement, pour la création de mondes sonores, un point de départ zéro? On a participé à un formidable atelier de sensibilité musicale. Assister au jaillissement sonore spontané dans l'attention la plus complète et non directive. Mario Bertoncini était notre maître zen du son.

En plus, il partageait avec nous, dans une grande générosité, sa vision actualisée de la composition. Le compositeur part du néant, l'idée musicale fait son chemin, impose ses exigences et commande la recherche des moyens. Au-delà de la série et de l'objet sonore, dorénavant perçus comme pièges de fétichisme et d'inauthenticité, l'ouverture et la spontanéité imposent 
de nouvelles œuvres, de véritables "sculptures sonores" taillées dans le matériau sonore. La notion même de tonalité musicale a encore une fois explosé, se recentrant autour de l'expressivité de la sonorité. Soit, une idée musicale pourrait encore nécessiter un quatuor à cordes, mais elle peut aussi bien imposer un montage de 500 klaxons de poids lourds...

Son utilisation des moyens électroacoustiques était également originale. II ne s'agissait pas de maîtriser un genre de musique défini, mais de fonctions à employer selon les besoins spécifiques d'une composition particulière. L'amplification pouvait servir, par exemple, comme un genre de microscope électronique, permettant l'intégration de sons microscopiques dans un contexte expressif. II nous a libérés du déterminisme du dernier appareil fourni par l'industrie. C'est le compositeur qui décide des fonctions techniques qui l'intéressent, pas le contraire.

"Source sonore", "sculpture sonore", "design musical" et "design sonore" étaient des termes nouveaux nous permettant de nous libérer, de nous situer et de nous ouvrir à des mondes musicaux nouveaux. La définition du design, à partir des travaux de mouvements comme celui du Bauhaus des années 1920, correspondait surtout à une notion visuelle. L'approche compositionnelle de Mario Bertoncini étendait cette notion à la dimension sonore. Il nous a sensibilisés à l'art de l'installation sonore. Vers la même époque, le Paysage sonore de R. Murray Schafer (The Tuning of the World, 1977) explorait et définissait le paysage sonore comme une énorme composition dont nous étions tous les auditeurs, interprètes et compositeurs. Dans un domaine connexe, le travail de création d'ambiances sonores cinématographiques s'est défini, à partir de la fin des années 1960, dans le nouveau métier de sound designer (concepteur sonore). La qualité (Dolby) et l'ampleur du champ sonore cinématographique multipiste généralisèrent la conscience de nouvelles possibilités sculpturales de création sonore.

Quelques-uns des étudiants de l'atelier de Mario Bertoncini ont fondé un groupe qui se nommait, à l'origine, MUD, employant de façon ironique un mot dérivé du terme Musical Design. On utilise avec ironie en anglais l'expression Our name is Mud! ("Notre nom est crotté!", pour signifier que l'on est mal vu.) Au moment du lancement d'un disque, le groupe s'est renommé Sonde et a continué à produire pendant près d'une dizaine d'années plus de 150 compositions collectives et événements publics. Les membres à l'origine étaient Andrew Culver, Keith Daniel, Pierre Dostie, Charles de Mestral, Chris Howard, Linda Pavelka; ils ont été rejoints, éventuellement, par Robin Minard et Michael O'Neill.

En un sens, vingt-huit ans plus tard, la bande sonore du film récent, Betty Goodwin : Le cœur à l'âme, 2003, par les anciens membres de Sonde, Pierre Dostie et Charles de Mestral, en est la plus récente création. Comment associer des musiques à des images déjà pleines de sens? Faut-il ajouter de la musique? Les images, fréquentes chez l'artiste, de corps humains flottant dans l'air ou dans l'eau ont servi de point de départ et guidé la recherche de la musique. Mario Bertoncini nous inspire encore, plus d'un quart de siècle plus tard. C'est la meilleure preuve de son influence, de sa grandeur d'âme... 
Avec le recul du temps, on se souvient particulièrement des séances de recherche improvisationnelles qu'il dirigeait avec un brio et un enthousiasme typiques et inimitables, ponctuées de surcroît d'anecdotes humoristiques sur, entre autres, le compositeur de musique de film Ennio Morricone, son ancien collègue à la Nuova Consonanza. Un développement inattentif, lors d'une improvisation, était interrompu bruyamment par un joyeux "Piece of shit!" ("Tas de merde!"). Un soir, il a dû manquer une des séances hebdomadaires de l'atelier. Ne pouvant pas y assister, il nous a laissé une note qui démontre sa générosité, sa lucidité, son enthousiasme et sa spontanéité.

La voici, en traduction :

Mes chers amis crottés,

Malheureusement, des AFFAIRES D'ÉTAT m'éloignent du champ de bataille ce soir.

Ne vous inquiétez pas, mon esprit sera présent, flottant et combattant le Mal musical de notre Faculté bien-aimée : la périodicité rythmique, les crescendos et cadences évidents, les motifs tonaux dans un contexte atonal. La symétrie, la prévisibilité et des milliers et des milliers d'autres monstruosités de l'Avant-garde officielle - précisément les armes qu'ELLE (l'Avant-garde) est habituée à employer dans le but de violer l'équilibre délicat de nos oreilles et de nos âmes. Alors, je souhaiterais que mon esprit contribue à nettoyer les fenêtres de notre atelier crotté!

Vale

Votre Paternel crotté

P.-S. : Souvenez-vous que la musique est aussi constituée de SILENCE.
My dear muddy friends!

Unfortunately STATE AFFAIRS will keep me out of the battlefield tonight.

Don't worry, my spirit will be there, floating around and fighting the Musical Evil of our beloved Faculty : rhythmical periodicity, crescendos and final obvious ending gestures, tonal rhythms within an atonal contexture, symmetry, predictabilty and thousands and thousands of other monstrosities of the currrent official Avant-garde - exactly the source of weapons with which SHE (the Avant-g) is used to violate the delicate balance of our ears and souls.

Alors! I wish my spirit could also contribute to cleaning the window of our muddy atelier.

Vale

Your Muddy old man

P.-S. : Please, do remember that music is also made of SILENCE.

Mario Bertoncini était le meilleur des maîtres, celui qui ne fait pas école, si le style ou la manière définissent l'école. II nous a montré comment être nous-mêmes, en cherchant à définir nos besoins dans une ouverture radicale. Il nous a permis d'être artistes à la fin du $x x^{e}$ siècle, un siècle qui a fait entrevoir l'éclatement de tout, de la syntaxe, de la perspective, de la tonalité artistique, de toutes les traditions culturelles, et même de la compassion humaine et de l'humanité, tout court. Compositeurs déboussolés ou enfants autochtones perdus de Davis Inlet, nous avons à relever le défi de la recherche du sens. Le passé n'est pas à renier mais l'avenir est un projet mené dans l'ouverture complète et l'attention absolue. 